\title{
Design on Automatic Spraying Robot System based on PC-PLC Network Control
}

Jian Li

Nanjing College of Chemical Technology, 210048, Nanjing, China

\begin{abstract}
With continuous industry upgrading and rapid economic transformation, all kinds of industrial robots are widely applied into various enterprises in China. In particular, important type of industrial robot (i.e. spray painting robot) is widely applied into the coating production process. Viewed from the current situation, however, it is very difficult for spray painting robot to be accepted by small and medium-sized enterprises. The main reason is that it is featured by relatively high price, strong technicality, difficulty in adapting to the production environment and weak reaction capacity toward the market. Therefore, it is necessary to develop a kind of universal type spray painting robot with low costs and realize network control.
\end{abstract}

Key words: PC-PLC, network control, automatic spraying robot system, design

With rapid development of national economy, practical improvement can be made to labor concentrated industry by practical development of high and new technology and improvement to the automation level of the manufacturing industry. This has already become the only route for China's economic transition and adjustment of industrial structure. A market foundation is laid for application and development of industrial robot by improving product quality, reducing cost investment and promoting the acceleration of market reaction. In this paper, the author states the design on automatic spray painting robot system based on PC-PLC network control.

\section{Development State of Spray Painting Robot at Home and Abroad}

In the end 1960s, Norwegian Trallfa Company invented the first spray painting robot in the world. Up to now, foreign countries have already walked through about 50 years in the development history of industrial robot spraying. They have relatively mature technologies. With continuous improvement to automatic spray painting robot in performance, function and other aspects at present, the spraying accuracy also reaches a very high level. Viewed from the world range, relatively famous spray painting robot manufacturers mainly include German KUKA, Japanese Yaskawa MOTOMAN and FANUC, French Staubli, Swiss ABB and American ADEPT. Among all these famous manufacturers, $\mathrm{ABB}$ is the most famous in the world. ABB has considerable valuable experiences in design and manufacturing of spray painting robot. Moreover, it also masters abundant spraying technologies. Therefore, its products enjoy wide development markets. In terms of the development of spray painting robot, China started relatively late. Moreover, China is still in the initial stage with slow development. Upon efforts for many years, however, 
China also made great progress in design and application of spray painting robot, no matter in theoretical research or in product research \& development. A complete system of robot design, development and application was gradually formed. However, there are lots of needs for design and application of small-sized spray painting robot in small-scale manufacturing enterprises.

\section{Research on Structure and Control Method of Spray Painting Robot}

(I) Mechanical structure of spray painting robot

Mechanical structure of spray painting robot is mainly used to meet outer elevation needs of spraying products. According to different structures, it is complex for shape of outer elevation of products. This proposes high requirements for flexibility of robot. Viewed from spraying accuracy, covering area of work and spraying flexibility, five-degree movable joint and mixed type mechanical structure should be adopted for spray painting robot. Mixed type robot is featured by such advantages as high work precision, stable performance and strong flexibility. In terms of spraying mode, rectangular coordinates are used to present three axes of $\mathrm{X}, \mathrm{Y}$ and $\mathrm{Z}$. The joint type pattern is of two axes: $\mathrm{P}$ and $\mathrm{R}$. Axis $\mathrm{X}$ is mainly used to track spraying object. Axis $\mathrm{Y}$ is used for spraying at the vertical direction. Axis $\mathrm{Z}$ is mainly responsible for adjusting the distance between spray gun and the object. Axes $\mathrm{P}$ and $\mathrm{R}$ are mainly used for all-dimensional and multi-angle locating of spray gun. The main body of robot utilizes the controller to drive the mechanical axis to work and promote its operation and make spray gun to reach the appointed spraying position.

\section{(II) Control structure of spray painting robot}

Mainly with PC-PLC as the core, the control system of spray painting robot includes object recognition, position control, safety protection, human-computer interface, network communication control, master control and operating control. The control object includes two parts: main body control and operating control over spraying object. At the same time, 5 mechanical axes are respectively controlled by 5 system drivers. Then, 5 systems are controlled with the pattern of high speed pulse dispatching by utilizing high speed pulse output and positioning control module of PLC. PC is mainly used to provide the operation interface of man-machine interaction, set up parameters, transmit control instructions and supervise system operation. PLC is the field controller of the system. It is mainly used to finish planning for transmission path of upper computer, control the implementation of site and speed control by 5-axis motor according to control instructions and feedback information of sensor and promote the motor to operate according to relevant requirements. Adjustment to the operating speed of spraying object is mainly decided by spraying technology. PLC is used to control the transducer and promote the motor to operate. Then, the spraying object is made to move stable by combining 
feedback information of the encoder. The system finishes connection between PC and PLC and realizes full combination of PC man-machine operation and PLC good control mainly through communication data line, so as to form the control process.

(III) Tracking and spraying object of spray painting robot

On industrial spraying field, assembly line operation is performed. The spraying object moves with the hanger in the form of pendant. Therefore, spray painting robot must perform synchronous movement with the spraying object. That is, motor is used to drive gears to move and finish the movement of pendant. However, the photoelectric encoder should finish the detection for operating speed of the motor. Then, the encoder feeds back obtained pulse to PLC. After that, PLC meters the number of pulse according to unit time and works out the operating frequency of motor. According to the operating frequency, PLC sends the tracked impulse frequency to Axis $\mathrm{X}$, so as to finish tracking of industrial robot for spraying object.

\section{Hardware Design of Automatic Spray Painting Robot Control System}

(I) Design of main circuit

Main circuit of spray painting robot is power supplied by a special power system, including UPS regulated power supply. It is used to provide power of various kinds of movable units. In the design process, the principle of guaranteeing power utilization safety of various modules should be embodied. Moreover, breaker should be arranged at positions with probably over current. Therefore, breaker should be added one by one for principal AC line and power supply of driver in main circuit of the system. At the same time, PLC is provided with a functional module to convert 220V AC to 24V DC. To effectively present PLC voltage instability caused by connection of several devices requiring $24 \mathrm{~V}$ DC to PLC, the functional module of converting $220 \mathrm{~V}$ AC to 24V DC should be added in the main circuit, so as to provide power to other functional modules requiring DC. PC-PLC network communication bus is used to collect real-time information and monitor and operate various parts of the robot system. For safety and controllability of the overall line and control over supply circuit switching of actuator, functional module of converting AC into DC and others, the motion control card is used to finish the above control. Power switch above the cabinet panel is used to control the action of motion control card. If the power switch is closed, the contactor will be powered on, and the entire circuit will be powered on accordingly.

(II) Design of control circuit

Control circuit of spray painting robot is designed based on the control thought of PC-PLC. It is featured by centralized operation, convenient configuration, flexible allocation, strong control ability and rich control algorithms. According to spraying requirements, continuous control, sequential control and batch processing control can be integrated together. Moreover, necessary 
special control algorithms can be conveniently added. Data is transmitted through communication network between operating points. The entire system shares information under coordinative operation, so as to obtain multi-function and optimized processing of the control system. Through configuration software, software and hardware are configured according to different spraying application objects, namely to determine connected relations between measurement and control signals, select proper control rules from the control algorithms library, deploy from the graphics library various monitoring and alarming pictures necessary for the formation of fundamental figures and constitute necessary control operating system.

Control circuit is mainly composed of basic module, input \& output module, communication module and driver module. As the control circuit, the functional orientation of input and output port is generally determined according to the value of each receiving and sending frequency. Input point mainly includes high speed input point and universal input point. The former is applicable to input of high-frequency signals such as encoder. High speed pulse input \& output point is mainly used to send impulse sequence and signal direction to driver. However, universal input point is used for starting \& stopping system and input of other switching values and switch signals of various axes. Universal output point is applied into braking and outputting control spray gun and system alarm switch.

\section{Software Design of Automatic Spray Painting Robot Control System}

Trapezoid modularization is mainly used as the design thought of software design on control system of spray painting robot. This software system is composed of 6 functional modules: system parameter initialization module, spraying object movement speed detection \& operation module, 5-axis origin restoration module, positive spraying module, left-side spaying module and right-side spraying module. The initialization module is mainly applied into zero clearing or negative value of temporary storage parameters, some auxiliary relays and other operations, such as the total number of spraying. The spraying object movement speed detection \& operation module includes two procedures: detection for movement frequency of spraying object and calculation of tracking frequency of robot. Generally, Axis X is used to track the object and provide data. The 5-axis origin restoration module is used for restoration procedure of returning various axes to original location at the starting link. The origin restoration of various axes makes it convenient for later route planning of robot. Front and left \& right spraying modules are mainly used to adjust coordination exercises of axes according to needs of corresponding modules. The six modules are made to alternative operation through special relay and calculator. Designed procedure is clear in logic and less in number of program.

Integration motion control instructions in PLC are used to adjust instructions and reach the 
purpose of controlling PLC pulse output mode. In case of special instructions, change is made to modulating pulse bandwidth PWM instruction of set value with effective features. The reason is that the sending period of this instruction input \& output pulse can be changed according to the set value. At the same time, the motor is adjusted to change the operating speed. It is extremely appropriate for Axis X whose change speed needs to be controlled at real time by the spraying object to be tracked. PLSR is an instruction attached with deceleration pulse output. Users can set speed and time within the specified area through this instruction, making it convenient for regulating the spraying time of speed axis and the tail spraying speed. As the moving speed of Axis $\mathrm{Y}$ is fast during spraying motion, generated noise is also great accordingly. However, this instruction can be used to effectively reduce the noise. The use of these instructions remarkably simplifies writing of programs and makes it more convenient for motor control.

\section{Conclusion:}

According to control design methods of the system, automatic spray painting robot can be used to spray relevant products and reach predicted requirements of tracking spraying. The design on automatic spray painting robot system based on PC-PLC network control is featured by low production costs and good system openness. It is the most appropriate to be widely applied and promoted in small and medium-sized spraying enterprises.

\section{Acknowledgments}

This paper is the 2012 Jiangsu province university "Blue Project" excellent young backbone teacher training project.

\section{References:}

[1] Xu Chengyi, Research on Planning and Off-line Programming of Industrial Robot Working Path [D], Nanjing: Nanjing Forestry University, 2006;

[2] Sun Xuejian, Yu Guohui, Zhou Wenqiao and Zhang Dapeng, Analysis on Development Characteristics of World Industrial Robot [J], Robot Technique and Application, 2008, (03): 8-9;

[3] Chen Yan, Yan Hua, Wang Liqiang and Hu Shixiong, Analysis on Uniformity of Robot Spraying Coating at Constant Speed [J], Journal of Tsinghua University (Science and Technology), 2010, (08): 1210-1213;

[4] Zheng Yuanguo and Xu Jianyun, Automatic Coating Machine based on PLC and Stepping Motor Control [J], Mechanical Engineering and Automation, 2011 (06): 49-50;

[5] Xiong Jun, Zhong Sidong and Mao Qingzhou, Detection of End Ultralimit of Freight Train with Light Curtain Measurement [J], Rail Vehicles, 2005, 43 (11): 35-37;

[6] Wang Jiahai and Wang Yi, Research on Route Optimization of White Body Welding Robot based on Ant Colony Algorithm [J], Manufacturing Automation, 2008, 30 (5): 16-17; 
[7] Zhou Bo, Qian Lai and Meng Zhengda, Route Sequence Optimization of Spray Painting Robot based on Ant Colony Algorithm [J], Computer Engineering, 2012, 38 (01);

[8] Jin Wenli, Yu Lili and Song Jian, Practical Method of PLC Pulse Output Stepping Motor [J], Mechanical \& Electrical Engineering Technology, 2006, 35 (08): 54-56;

[9] Zhao Dean, Chen Wei and Tang Yang, Planning for Spray Gun Route of Spray Painting Robot based on Genetic Algorithm [J], China Mechanical Engineering, 2008, 19 (07): 777-779;

[10] Feng Chuan and Sun Zengqi, Spray Torch Modeling and Simulation Research in Robot Spraying Process [J], Robot, 2009, 25 (04): 353-358. 